\title{
Essais de fractionnement de la caséine humaine Analyse de ses constituants
}

\author{
par \\ Evelyne CLEMENT, C. ALAIS \\ Laboratoire de Biochimie Appliquée, Université de Nancy-I \\ et P. JOLLES \\ Laboratoire des Protéines, Université de Paris-V
}

\section{INTRODUCTION}

Les caséines des espèces monogastriques ont été beaucoup moins étudiées que celles des ruminants. Toutes sont constituées de mélanges complexes de protéines; les caséines des ruminants forment une famille qui paraît être homogène, avec quatre groupes de constituants $\alpha . s, \beta, \chi$ et $\gamma$; les caséines des non-ruminants sont très différentes dans la variété de leur constitution.

Plusieurs équipes de chercheurs ont essayé de fractionner la caséine humaine. Malpress et al. (1964, 1966), Alais et Jollès (1964, 1969, 1970), Nagasawa et al. (1967, 1969, 1970, 1971, 1974), Groves et al. (1970), Toyoda et Yamauchi (1973) ont obtenu des fractions plus ou moins pures, mais on relève des contradictions notables dans l'interprétation des résultats, en ce qui concerne les constituants sensibles au calcium (comme les caséines $\alpha_{\mathrm{s}}$ et $\beta$ bovines) et le, ou les constituants insensibles au calcium et sensibles à la chymosine (comme la caséine $\chi$ bovine), et aussi dans les résultats analytiques (teneur en phosphore et en acide sialique). Le point commun à ces recherches est l'hétérogénéité de la caséine humaine. On retire l'impression d'une étude bibliographique que les chercheurs n'ont pas travaillé sur la même substance.

Toutes ces constatations sur l'hétérogénéité de cette protéine ont permis à Malpress et Hytten (1964) d'émettre l'hypothèse que la caséine humaine aurait des propriétés variables, dépendant de l'activité enzymatique de la glande mammaire, et de la composition du

35e communication sur les caséines. 
lait dont elle provient. Ces auteurs, ainsi que Alais et Jollès (1970) constatent une évolution de la composition électrophorétique avec le stade de lactation ; on assisterait à un " mûrissement » de cette protéine.

Les nouveaux essais de fractionnement que nous décrivons dans cette communication, se placent dans l'axe de ceux exposés précédemment par Alais et Jollès (1969). Diverses techniques de chromatographie et d'électrophorèse préparative ont été utilisées. Outre l'analyse chimique des fractions, leur sensibilité à la chymosine (EC 3.4.23.4) a été éprouvée.

\section{MATERIEL ET METHODES}

\section{1) Préparation des caséines}

Les caséines humaines que nous avons utilisées ont été préparées à partir de laits de mélange ou de laits individuels. Ces laits proviennent soit de la région de Nancy, soit de la région parisienne.

Nous avons suivi la technique préconisée par Alais et Jollès (1970). Le lait, exempt de colostrum, est écrémé par centrifugation à $4^{\circ} \mathrm{C}$; il est ensuite dialysé à $2^{\circ} \mathrm{C}$ pendant plusieurs jours ; la caséine est précipitée par $\mathrm{HCl} 1 \mathrm{M}$ jusqu'à $\mathrm{pH} 4,6$; elle est lavée quatre fois à l'eau, deux fois avec de l'éthanol 95 p. 100 puis avec de l'éther éthylique ; enfin, elle est séchée par lyophilisation.

Nous avons disposé de vingt échantillons de caséine. Avant de commencer les fractionnements par chromatographie, nous avons examiné leur comportement électrophorétique et leur sensibilité vis-àvis de la chymosine.

\section{2) Fractionnement}

\subsection{Chromatographie}

La plupart des auteurs qui ont travaillé sur le fractionnement de la caséine humaine ont utilisé la chromatographie sur colonne de DEAE-cellulose. Nous avons à nouveau utilisé ce support, mais nous avons abandonné le tampon phosphate contenant de la diméthylformamide employé précédemment par Alais et Jollès (1969). Nous avons tout d'abord appliqué le système recommandé par Groves et Gordon (1970) qui comporte des tampons phosphate à $\mathrm{pH} 6,5$ de différentes forces ioniques, le dernier contenant $4 \mathrm{M}$ d'urée ; ensuite, nous avons fait l'essai du tampon Tris- $\mathrm{HCl}$ de $\mathrm{pH} 8,6$, contenant 3,3 M d'urée et 0,3 p. 100 de mercaptoéthanol, avec gradient de molarité de $\mathrm{NaCl}$ 0-0,2 M utilisé par Testud et Ribadeau-Dumas (1973) pour fractionner la caséine de lapine. En outre, des essais de purification des fractions par filtration sur gel de Séphadex G-100 ont été exécutés avec ce dernier tampon. 


\subsection{Electrophorèse préparative}

Cette technique a été employée pour une seconde séparation des fractions isolées par chromatographie. On a utilisé la colonne Shandon avec différentes hauteurs de gel de polyacrylamide à 7,5 p. 100 contenant $6 \mathrm{M}$ d'urée à $\mathrm{pH} 9,5$; le tampon d'électrode est composé de Tris-glycine à $\mathrm{pH} 8,3$ et le tampon d'élution de Trisacide acétique à $\mathrm{pH} 8,0$.

\section{3) Méthodes analytiques}

L'analyse électrophorétique des fractions a été effectuée sur gel d'amidon en milieu urée $7 \mathrm{M}$ à pH 8,6 selon Wake et Baldwin (1961).

Le phosphore a été dosé, après digestion acide, par la méthode colorimétrique de Bamann et al. (1948) et l'azote par microKjeldahl.

Les oses neutres ont été dosés par la méthode à l'orcinol, selon Schultze et al. (1958) après hydrolyse par $\mathrm{HCl} 1 \mathrm{~N}, 3 \mathrm{~h}$ à $100^{\circ} \mathrm{C}$; les osamines, par le réactif d'Ehrlich selon Rondle et Morgan (1955), après hydrolyse par $\mathrm{HCl} 2 \mathrm{~N}, 3 \mathrm{~h}$ à $100^{\circ} \mathrm{C}$ et les acides sialiques, par la méthode à l'acide thiobarbiturique selon Warren (1959) après hydrolyse par $\mathrm{H}_{2} \mathrm{SO}_{4} 0,1 \mathrm{~N}, 1 \mathrm{~h}$ à $85^{\circ} \mathrm{C}$.

La détermination des acides aminés a été faite au moyen de l'autoanalyseur Technicon selon la technique de Piez et Morris (1960) après hydrolyse acide $\left(\mathrm{HCl} 6 \mathrm{~N}, 110^{\circ} \mathrm{C}\right.$ ) durant $18 \mathrm{~h}, 48 \mathrm{~h}$ et $72 \mathrm{~h}$.

La sensibilité à la chymosine a été déterminée en appliquant la méthode de Alais et al. (1953) ; l'azote non protéique (soluble dans 12 p. 100 d'acide trichloracétique) a été dosé par micro-Kjeldahl, après $30 \mathrm{mn}$ de digestion à $30^{\circ} \mathrm{C}$ d'une solution à $1 \mathrm{p}$. 100 et à $\mathrm{pH} 6,7$; la quantité d'enzyme cristallisée utilisée est de $0,7 \mu \mathrm{g} / \mathrm{ml}$.

La sensibilité au calcium a été montrée par l'apparition d'un précipité lorsque l'on ajoute $0,03 \mathrm{M}$ de chlorure de calcium dans la solution à 1 p. 100 et $\mathrm{pH} 6,7$.

L'absorption spécifique des constituants séparés est mesurée à $280 \mathrm{~nm}$ au moyen de spectrophotomètre Acta II.

\section{RESULTATS}

\section{1) Comportement électrophorétique des caséines humaines}

Les migrations électrophorétiques en gel d'amidon à $\mathrm{pH} 8,6$ de dix-sept échantillons de caséines humaines d'origines variées, individuelles ou de mélange, avec des dépôts d'une quantité invariable de protéines révèlent des différences importantes dans le nombre et la disposition des constituants. Dans le cas général, la caséine humaine précipitée à $\mathrm{pH}$ 4,6 se présente ainsi : bandes lentes souvent précédées 
d'une traînée, bandes moyennes plus nombreuses que dans les deux autres zones et bandes rapides d'intensités différentes. Mais on note l'absence de bandes chez certaines caséines ou des bandes qui migrent différemment. La figure 1 présente cinq exemples caractéristiques de

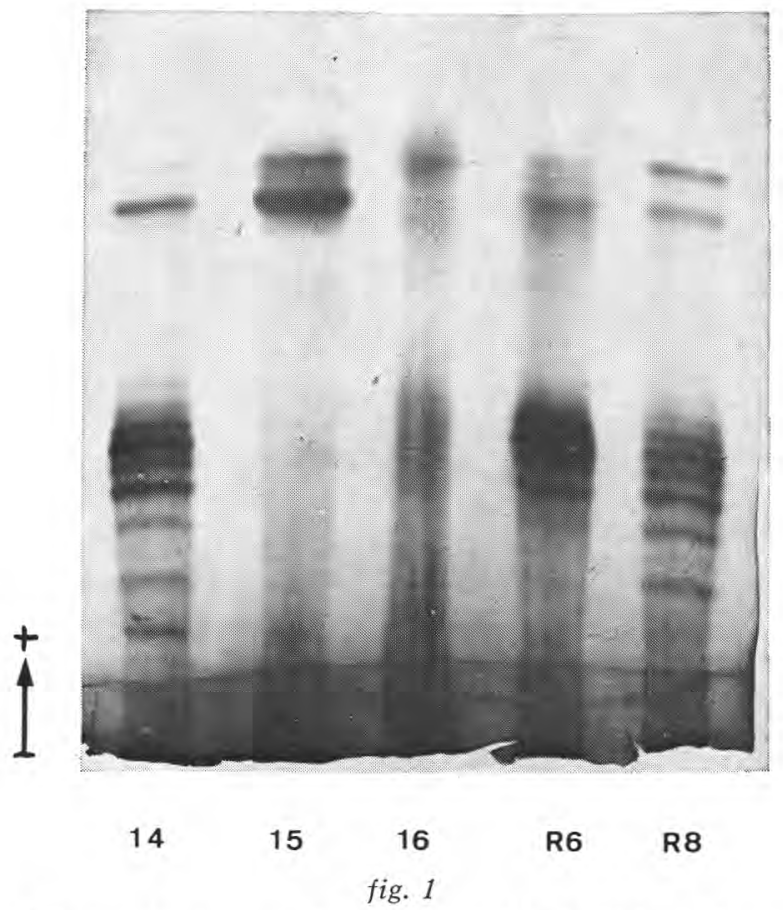

Electrophorèse en gel d'amidon à pH 8,6 d'échantillons de caséine humaine

migrations ; les échantillons 14, R6 et R8 donnent des diagrammes comparables, avec de nombreuses bandes. L'échantillon 15 est constitué surtout de bandes rapides. L'échantillon 16 donne un diagramme confus, la substance est peu soluble.

Pour préciser les positions, nous avons pris comme unité de migration une bande rapide, nette et toujours présente, parfois précédée d'une bande fine dont la mobilité serait de 1,02. Les bandes moyennes ont une mobilité relative proche de 0,4 ou 0,5 et les bandes lentes, lorsqu'elles sont clairement visibles, ce qui n'est pas toujours le cas, sont dans la zone de 0,2-0,3. Dans des conditions identiques d'examen, les mobilités des différents groupes de bandes ne sont pas exactement les mêmes d'une caséine à l'autre. 
A $\mathrm{pH} 3,8$, l'ordre des constituants rapides, moyens ou lents reste le même ; les bandes sont moins nettes, mais elles sont plus nombreuses qu'à $\mathrm{pH} \mathrm{8,6}$. L'électrophorèse sur disques de polyacrylamide à 7 p. 100 donne la même image que le gel d'amidon à $\mathrm{pH}$ 8,6.

Les différences notables observées au cours de l'examen électrophorétique des caséines pose le problème important du choix des échantillons pour les opérations de fractionnement. Nous avons retenu de préférence les préparations de caséine comportant le plus grand nombre de bandes, en supposant que ce soient les plus normales.

\section{2) Sensibilité à la chymosine}

Tous les échantillons de caséines humaines ont été analysés pour leur taux d'azote non protéique (NPN) soluble dans l'acide trichloracétique, avant et après action de la chymosine.

\section{TABLEAU 1}

Action de la chymosine sur la caséine humaine

\begin{tabular}{|c|c|c|}
\hline \multirow{2}{*}{$\begin{array}{l}\text { Caséine* } \\
\text { (solution à } 0,2 \text { p. 100) }\end{array}$} & \multicolumn{2}{|c|}{ Azote non protéique p. $100 \mathrm{~N}$ total } \\
\hline & Avant digestion & $\begin{array}{l}\text { Augmentation } \\
\text { après digestion }\end{array}$ \\
\hline $1(\mathrm{~m})$ & & \\
\hline $2(\mathrm{~m})$ & $\begin{array}{l}1,23 \\
7,92\end{array}$ & $\begin{array}{l}0,49 \\
0\end{array}$ \\
\hline $3(\mathrm{~m})$ & 6,76 & 0,02 \\
\hline 4 (i) & 11,82 & 0,02 \\
\hline $5(\mathrm{i})$ & 5,37 & 3,68 \\
\hline 6 (i) & 7,16 & 1,89 \\
\hline 7 (i) & 11,61 & 2,15 \\
\hline $8(\mathrm{~m})$ & 6,6 & 2,67 \\
\hline 9 (i) & 9,59 & 0 \\
\hline 10 (i) & 5,01 & 0,01 \\
\hline $11(\mathrm{~m})$ & 8,44 & 3,43 \\
\hline 12 (i) & 4,66 & 0,77 \\
\hline $13(\mathrm{~m})$ & 9,8 & 0,5 \\
\hline $14(\mathrm{~m})$ & 5,89 & 0,19 \\
\hline 15 (i) & 4,89 & 2,59 \\
\hline $16(\mathrm{~m})$ & 7,46 & 0,03 \\
\hline R6 (m) & 2,90 & 3,06 \\
\hline $\mathrm{R} 8(\mathrm{~m})$ & 16,72 & 0,06 \\
\hline $\mathrm{R} 9(\mathrm{~m})$ & 1,88 & 0,49 \\
\hline
\end{tabular}

* $\mathrm{i}$ = lait individuel $; \mathrm{m}=$ lait de mélange.

Les échantillons « $\mathrm{R}$ " proviennent de laits de la région parisienne. 
TABLEAU 2. - Essais de fractionnements des caséines humaines par chromatographie

\begin{tabular}{|c|c|c|c|c|c|c|}
\hline Caséine & $\begin{array}{c}\text { Essai } \\
n^{\circ}\end{array}$ & Support & Tampon de départ & Palier $(P)$ ou gradient $(G)$ & $\begin{array}{c}\text { Charge } \\
\mathrm{g}\end{array}$ & $\begin{array}{c}\text { Colonne } \\
\mathrm{cm}\end{array}$ \\
\hline R6 & 1 & DEAE-cellulose & Phosphate $0,005 \mathrm{M} \mathrm{pH} \mathrm{8,3}$ & $\begin{array}{l}\text { (P) phosphate } 0,1 \mathrm{M} \text { pH } 6,5 \mathrm{NaCl} 0,3 \mathrm{M} \\
\text { (P) phosphate } 0,1 \mathrm{M} \text { pH 6,5 urée } 4 \mathrm{M}\end{array}$ & 1 & $2,5 \times 50$ \\
\hline R6 & 2 & DEAE-cellulose & Phosphate $\mathrm{pH} 8,30,005 \mathrm{M}$ & $\begin{array}{l}\text { (G) phosphate } 0,005-0,1 \mathrm{M} \\
\text { (P) } 0,1 \mathrm{M} \text { pH } 6,5 \mathrm{NaCl} 0-0,3 \mathrm{M} \\
\text { (P) } 0,1 \mathrm{M} \text { pH } 6,5 \text { urée } 4 \mathrm{M}\end{array}$ & 1 & $2,5 \times 50$ \\
\hline 14 & 3 & DEAE-cellulose & Phosphate $0,005 \mathrm{M} \mathrm{pH} 8,3$ & $\begin{array}{l}\text { (P) phosphate } 0,1 \mathrm{M} \text { pH } 6,5 \mathrm{NaCl} 0,3 \mathrm{M} \\
\text { (P) phosphate } 0,1 \mathrm{M} \text { pH } 6,5 \text { urée } 4 \mathrm{M}\end{array}$ & 1 & $2,5 \times 50$ \\
\hline 4 & 4 & DEAE-cellulose & Phosphate $0,005 \mathrm{M} \mathrm{pH} \mathrm{8,3}$ & $\begin{array}{l}\text { (P) phosphate } 0,1 \mathrm{M} \mathrm{pH} 6,5 \mathrm{NaCl} 0,3 \mathrm{M} \\
\text { (P) phosphate } 0,1 \mathrm{M} \mathrm{pH} 6,5 \text { urée } 4 \mathrm{M}\end{array}$ & 0,5 & $2,5 \times 50$ \\
\hline $\begin{array}{l}\text { Fraction } n^{\circ} 4: \\
\text { - rapides } \\
\text { - lentes } \\
\text { - rapides et moyennes } \\
\text { - lentes et moyennes }\end{array}$ & $\begin{array}{l}5 \\
6 \\
7 \\
8\end{array}$ & $\begin{array}{l}\text { DEAE-cellulose } \\
\text { DEAE-cellulose } \\
\text { DEAE-cellulose } \\
\text { CM cellulose }\end{array}$ & $\begin{array}{l}\text { Phosphate } 0,1 \mathrm{M} \text { pH } 7,5 \\
\text { Phosphate } 0,1 \mathrm{M} \mathrm{pH} \mathrm{7,5} \\
\text { Phosphate } 0,1 \mathrm{M} \mathrm{pH} 7,5 \\
\text { Acétate } 0,5 \mathrm{M} \mathrm{pH} 5,0\end{array}$ & $\begin{array}{l}\text { (G) } \mathrm{NaCl} 0-0,5 \mathrm{~N} \\
\text { (G) } \mathrm{NaCl} 0-0,5 \mathrm{~N} \\
\text { (G) } \mathrm{NaCl} 0-0,3 \mathrm{~N} \\
\text { (G) } \mathrm{NaCl} 0-0,5 \mathrm{~N}\end{array}$ & $\begin{array}{l}0,530 \\
0,125 \\
0,125\end{array}$ & $\begin{array}{l}2,5 \times 50 \\
2,5 \times 50 \\
0,75 \times 30\end{array}$ \\
\hline R8 & 9 & DEAE-cellulose & Tris-urée 3,3 M pH 8,6 & (G) $\mathrm{NaCl} 0-0,3 \mathrm{~N}(2 \mathrm{l})$ & 1 & $2,5 \times 50$ \\
\hline $\mathrm{R} 8$ & 10 & DEAE-cellulose & Tris-urée 3,3 M pH 8,6 & (G) $\mathrm{NaCl} 0-0,35(2 \mathrm{l})$ & 1 & $2,5 \times 80$ \\
\hline $\begin{array}{l}\text { Fractions de R } 8 \text { : } \\
\text { - lentes } \\
\text { - moyennes } \\
\text { - rapides }\end{array}$ & $\begin{array}{l}11 \\
12 \\
13\end{array}$ & $\begin{array}{l}\text { DEAE-cellulose } \\
\text { Séphadex G-100 } \\
\text { Séphadex G-100 }\end{array}$ & $\begin{array}{l}\text { Tris-urée } 3,3 \mathrm{M} \mathrm{pH} \mathrm{8,6} \\
\text { Tris-urée 3,3 M pH 8,6 } \\
\text { Tris-urée 3,3 M pH 8,6 }\end{array}$ & (G) $\mathrm{NaCl} 0-0,35 \mathrm{~N}(0,51)$ & $\begin{array}{l}0,053 \\
0,31 \\
0,44\end{array}$ & $\begin{array}{l}2,5 \times 35 \\
2,5 \times 100 \\
2,5 \times 100\end{array}$ \\
\hline
\end{tabular}


D'après le tableau 1 , nous constatons de grandes différences quand on compare les pourcentages de l'azote soluble dans l'acide trichloracétique avant digestion enzymatique ; la solubilité des caséines dans ce réactif varie dans le rapport de 1 à 8 environ. Cette variabilité se retrouve après action de la chymosine. Sept caséines ne présentent pas ou très peu d'augmentation de NPN. Par contre, six caséines donnent des augmentations plus élevées que la caséine bovine (plus de 2 p. 100).

\section{3) Essais de fractionnement par chromatographie sur DEAE- cellulose}

Nous avons retenu quatre échantillons de caséine ; l'image électrophorétique de trois d'entre eux se trouve sur la figure 1 (14, R6, R8). L'ensemble des essais de fractionnement est présenté succinctement dans le tableau 2.

Les essais $n^{\circ} 1,3$ et 4 comportent une élution par trois tampons phosphate de composition différente, dans les conditions indiquées par les auteurs ayant publié les essais de fractionnement les plus récents (Groves et Gordon, 1970).

Les résultats varient quelque peu selon la caséine mise en œuvre. La caséine R6 (essai $n^{\circ} 1$ ) se résoud en quatre pics ; aucun ne correspond à une substance électrophorétiquement homogène. Une modification de la technique d'élution, avec un gradient de molarité de phosphate (essai 2) augmente le nombre de fractions jusqu'à 9, mais leur composition électrophorétique n'est pas satisfaisante. La caséine 14, dans les conditions de l'essai 1, donne sept fractions également hétérogènes. Par contre, la caséine 4 (essai 4) qui provient d'un lait individuel, donne un profil chromatographique analogue au précédent, mais avec une meilleure séparation des constituants. La figure 2 montre qu'un constituant de mobilité moyenne est séparé dans le tampon de départ et que les deux constituants rapides sont séparés des constituants moyens et lents par le tampon $\mathrm{pH}$ 6,5 contenant $\mathrm{NaCl}$.

Les fractions séparées dans l'essai 4 (fig. 2) et dans des essais parallèles, ont été rechromatographiées sur le même support, avec un tampon phosphate plus concentré et un $\mathrm{pH}$ un peu plus bas, suivi d'un gradient de $\mathrm{NaCl}$. La figure 3 présente le début de la chromatographie de la fraction rapide ( $n^{\circ} 5$ ) ; le tampon phosphate a permis de séparer deux constituants rapides, exempts de constituants lents. La rechromatographie des constituants lents et moyens dans les mêmes conditions n'a pas permis une séparation complète ; mais il faut signaler que l'électrophorèse a révélé des bandes migrant plus rapidement qu'à l'origine.

Un nouvel essai de séparation des constituants lents et moyens a été fait sur un support acide, la carboxy-méthylcellulose ( $n^{\circ} 8$, tab. 2) : il a été négatif, les pics séparés correspondant à un mélange. 

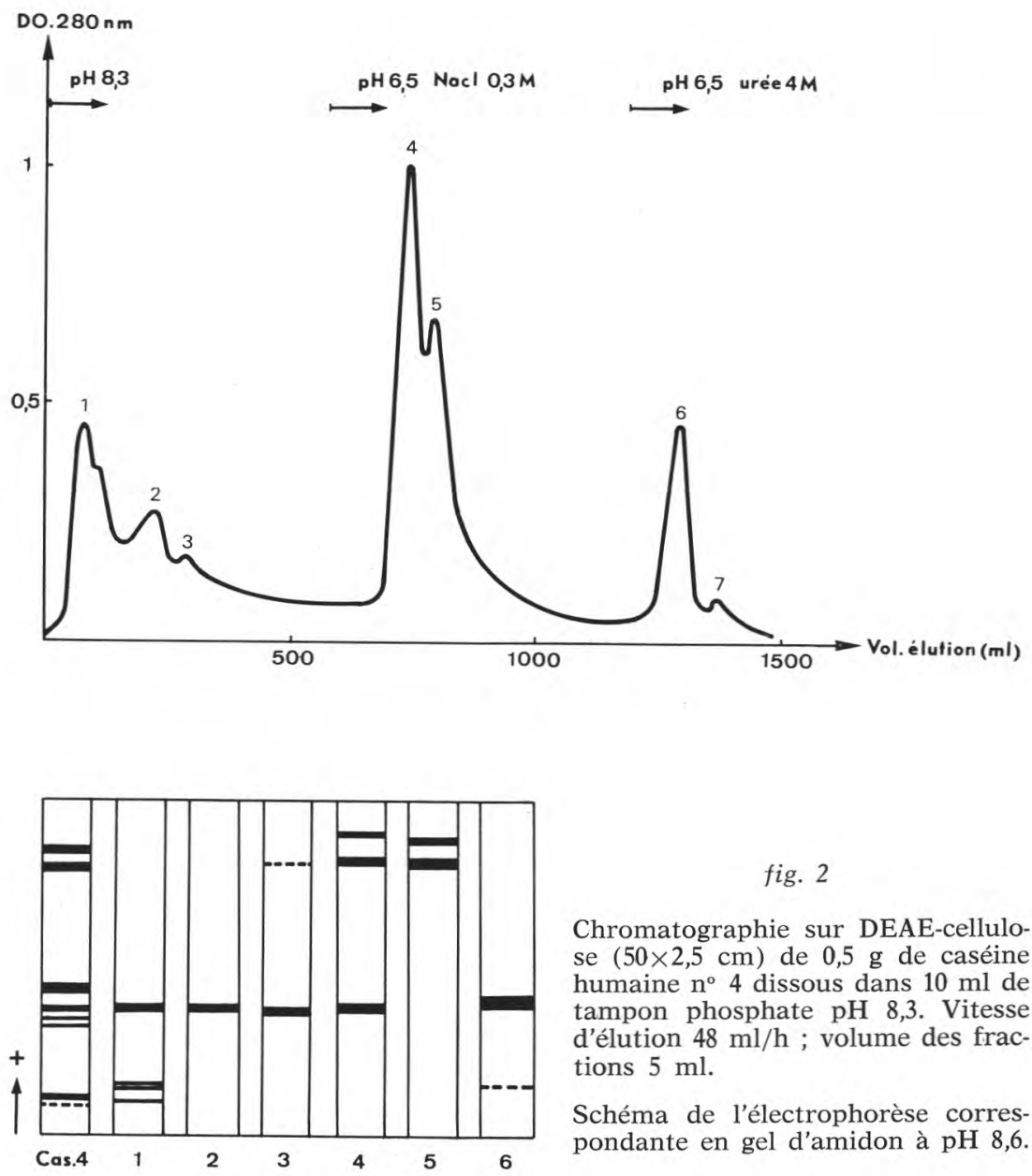

fig. 2

Chromatographie sur DEAE-cellulose $(50 \times 2,5 \mathrm{~cm})$ de $0,5 \mathrm{~g}$ de caséine humaine $\mathrm{n}^{\circ} 4$ dissous dans $10 \mathrm{ml}$ de tampon phosphate $\mathrm{pH} 8,3$. Vitesse d'élution $48 \mathrm{ml} / \mathrm{h}$; volume des fractions $5 \mathrm{ml}$.

Schéma de l'électrophorèse correspondante en gel d'amidon à $\mathrm{pH} 8,6$.

Des essais de fractionnement sur des colonnes plus longues et avec un autre tampon (Tris-Urée) plus alcalin que les précédents $(\mathrm{pH} 8,6)$ et une autre caséine $(\mathrm{R} 8)$ ont permis de séparer à nouveau des fractions lentes, des fractions rapides et des fractions moyennes dans les proportions relatives de 2, 55 p. 100 et 43 p. 100 environ ; mais chacune se révèle hétérogène par l'électrophorèse. Le groupe des fractions lentes a été rechromatographié dans les mêmes conditions ; nous n'avons pas obtenu la séparation complète des bandes qui le composent, mais nous avons vu apparaître, dans un pic élué par le gradient de $\mathrm{NaCl}$, des bandes plus rapides que nous n'avions pas observées dans la fraction d'origine. 

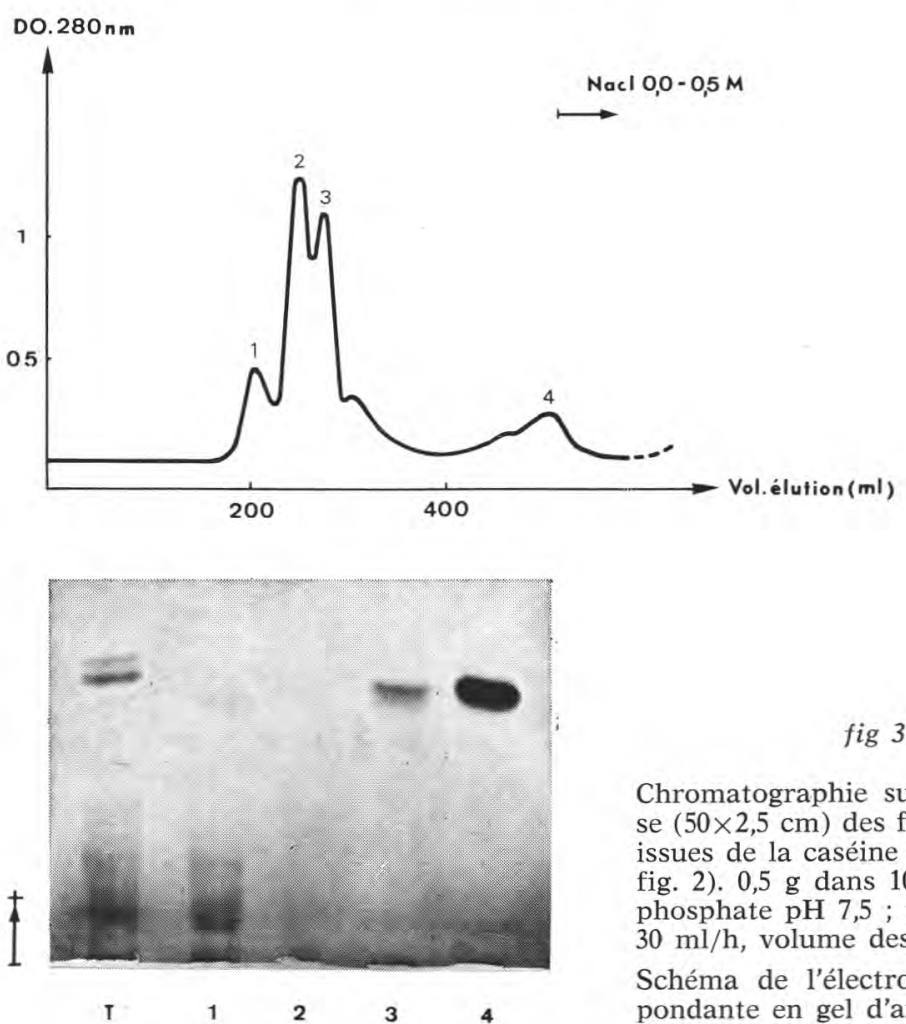

fig 3

Chromatographie sur DEAE-cellulose $(50 \times 2,5 \mathrm{~cm})$ des fractions rapides issues de la caséine $\mathrm{n}^{\circ} 4$ (fraction 5 , fig. 2). $0,5 \mathrm{~g}$ dans $10 \mathrm{ml}$ de tampon phosphate $\mathrm{pH} 7,5$; vitesse d'élution $30 \mathrm{ml} / \mathrm{h}$, volume des fractions $5 \mathrm{ml}$. Schéma de l'électrophorèse correspondante en gel d'amidon à $\mathrm{pH} 8,6$.

Les fractions moyennes et rapides, issues des essais avec le tampon Tris-Urée, ont été filtrées sur Séphadex G-100 ; on a ainsi pu séparer un constituant moyen qui est homogène à l'électrophorèse à pH 8,6 en gel d'amidon (essai 12).

\section{4) Electrophorèse préparative sur colonne}

Des essais ont été effectués avec la caséine R8 et différentes hauteurs de gel de polyacrylamide : 5,7 et $14 \mathrm{~cm}$ à $\mathrm{pH} 8,3$. On observe, au cours de l'élution, un premier pic non protéique au front de migration ; ensuite, viennent les fractions rapides, puis les fractions moyennes ; les fractions lentes n’ont pas été retrouvées. La figure 4 montre le diagramme d'élution avec la colonne de $14 \mathrm{~cm}$. Le fractionnement prend l'allure d'une répartition régulière de constituants selon leur charge, comme si des modifications des constituants originaux avaient eu lieu, le constituant $n^{\circ} 2$ le plus rapide ayant une 

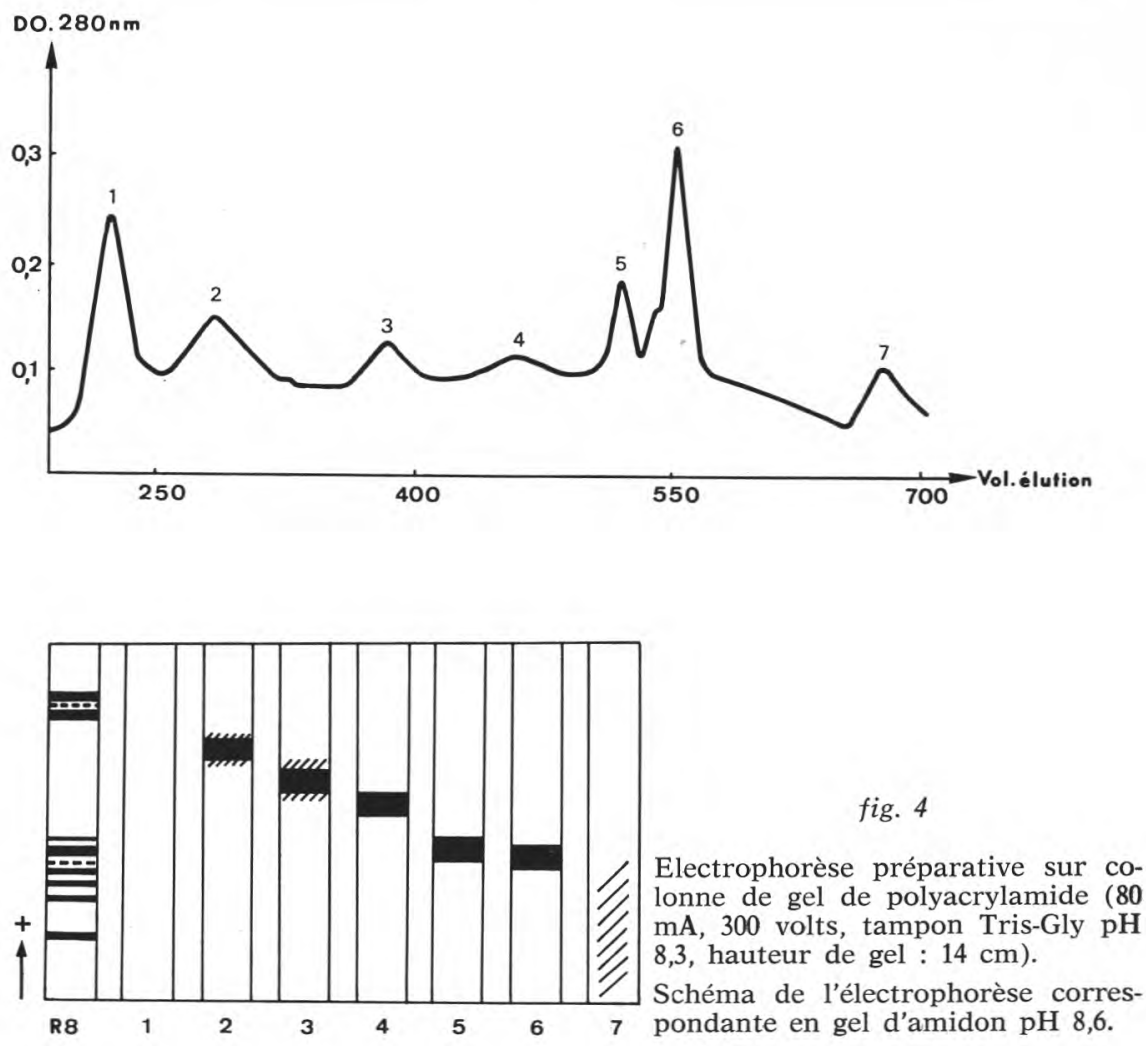

mobilité nettement inférieure à celle du groupe rapide de la caséine entière.

\section{5) Propriétés et composition des fractions}

Le tableau 3 résume quelques propriétés des fractions de la caséine humaine. La fraction rapide possède les deux caractéristiques essentielles de la caséine $\chi$ des ruminants : sensibilité à la chymosine à $30^{\circ} \mathrm{C}$ et insensibilité au calcium (non précipitée en présence de $0,03 \mathrm{M} \mathrm{CaCl}_{2}$ ) ; les deux autres fractions se comportent comme les caséines $\alpha_{\mathrm{s}}$ et $\beta$.

Toutes ces fractions contiennent des glucides, mais en proportions différentes. On note que la fraction rapide ne contient pas d'acide sialique, contrairement à la caséine $\chi$ bovine. La teneur en phosphore croît avec la mobilité.

Le tableau 4 présente la composition en acides aminés de trois constituants homogènes à l'électrophorèse à $\mathrm{pH}$ 8,6 : un constituant 
TABLEAU 3

Propriétés des fractions et composition de la partie non peptidique

\begin{tabular}{l|c|c|c}
\hline & \multicolumn{2}{|c}{ Fractions } \\
\cline { 2 - 3 } & Lente & Moyenne & Rapide \\
\cline { 2 - 3 } & & & \\
\hline & & & \\
Sensibilité à la chymosine* & 0 & 0 & 5,2 \\
Sensibilité au calcium & + & + & - \\
Absorption spécifique & 0,724 & 0,968 & 1,35 \\
(280 nm, 1 cm, 1 mg/ml) & 12,08 & 12,94 & 15,42 \\
Azote p. 100 & 0,08 & 0,38 & 0,50 \\
Phosphore p. 100 & 4,21 & 1,36 & 1,92 \\
Hexoses réducteurs p. 100 & 6,55 & 1,25 & 0,95 \\
Hexosamines (N-acétylés) p. 100 & 1,42 & 0,14 & 0 \\
Acide sialique** p. 100 & & & \\
& & & \\
\hline
\end{tabular}

* Augmentation de l'azote non protéique p. 100 de l'azote total après digestion.

** En acide $\mathrm{N}$-acétylneuraminique.

moyen et deux constituants rapides ( $R_{1}$ est le plus mobile). Mais il faut signaler qu'au cours de l'action de la carboxypeptidase sur le constituant moyen, plusieurs acides aminés sont libérés : la valine est prédominante. Le constituant moyen paraît dépourvu de cystéine et il est riche en proline (comme les caséines $\alpha_{\mathrm{s}}$ et $\beta$ bovines). Au contraire, les constituants rapides contiennent de la cystéine et beaucoup moins de proline ; ils présentent entre-eux des analogies.

\section{DISCUSSION}

La caséine humaine, précipitée à $\mathrm{pH}$ 4,6 est une substance dont les comportements électrophorétiques et chromatographiques, la solubilité dans l'acide trichloracétique et la sensibilité à la chymosine varient d'un échantillon à l'autre. Elle se différencie ainsi de la caséine des ruminants qui présente une grande régularité, en dehors de la période colostrale.

Des variations de cet ordre avaient déjà été observées par Alais et Jollès (1969). Evidemment, l'espèce humaine ne peut présenter l'uniformité constatée chez les ruminants, qui ont subi une sélection volontaire. La grande variabilité observée pourrait résulter de certaines dégradations de constituants fragiles au cours des traitements 


\section{TABLEAU 4}

Composition en acides aminés de trois constituants de la caséine humaine (p. $100 \mathrm{~g}$ de substance sèche)

\begin{tabular}{|c|c|c|c|}
\hline & \multicolumn{3}{|c|}{ Constituants } \\
\hline & Moyen & Rapide R1 & Rapide R2 \\
\hline $\begin{array}{l}\text { Acide aspartique } \\
\text { Thréonine } \\
\text { Sérine } \\
\text { Acide glutamique } \\
\text { Proline } \\
\text { Glycine } \\
\text { Alanine } \\
\text { Valine } \\
\text { Cystéine } \\
\text { Méthionine } \\
\text { Isoleucine } \\
\text { Leucine } \\
\text { Tyrosine } \\
\text { Phénylalanine } \\
\text { Lysine } \\
\text { Histidine } \\
\text { Arginine }\end{array}$ & $\begin{array}{r}5,9 \\
3,1 \\
2,7 \\
21,2 \\
16,6 \\
0,7 \\
2,2 \\
8,6 \\
1,9 \\
7,1 \\
10,2 \\
3,9 \\
2,7 \\
4,7 \\
2,3 \\
2,9\end{array}$ & $\begin{array}{r}14,5 \\
4,4 \\
5,7 \\
18,7 \\
3,9 \\
2,8 \\
2,7 \\
2,4 \\
3,2 \\
1,1 \\
8,6 \\
8,6 \\
3,7 \\
2,9 \\
9,7 \\
3,6 \\
1,6\end{array}$ & $\begin{array}{r}12,4 \\
3,9 \\
4,9 \\
20,7 \\
9,5 \\
2,8 \\
2,4 \\
3,3 \\
0,8 \\
0,5 \\
7,0 \\
6,2 \\
4,4 \\
3,0 \\
8,5 \\
5,1 \\
3,3\end{array}$ \\
\hline
\end{tabular}

ou d'un début de protéolyse dans le lait humain qui est souvent riche en germes microbiens.

Les divergences dans les résultats obtenus par le petit nombre d'auteurs ayant publié des expériences de fractionnement (Malpress et Seid-Akhavan (1966), Alais et Jollès (1969), Nagasawa et al. (1967, 1970), Groves et al. (1970), Toyoda et Yamauchi (1973) sont décourageantes. Les facteurs raciaux et alimentaires sont certainement à considérer ; ils s'ajoutent aux différences dans les techniques employées pour la préparation et la purification.

La chromatographie nous a permis de séparer trois groupes renfermant chacun de deux à quatre constituants. A partir d'une caséine de lait individuel, nous avons isolé les deux constituants du groupe le plus mobile à l'électrophorèse à $\mathrm{pH} 8,6$; cette purification n'a pas pu être renouvelée à partir de laits de mélange de lactarium. Un constituant de mobilité moyenne a également été isolé de plusieurs échantillons.

Nous nous sommes heurtés à une difficulté inexpliquée : après des rechromatographies et des électrophorèses préparatives on observe 
des déplacements de bandes dans l'électrophorèse en gel. Des modifications de charge doivent se produire ; elles sont peut-être consécutives à une libération de phosphore labile ou d'acide sialique.

Groves et Gordon (1970) pensent que la caséine humaine est constituée de six fractions identiques dans leur partie peptidique et diversement phosphorylée, d'un poids moléculaire de 25000 environ. Nous avons observé comme ces auteurs, que la teneur en phosphore augmentait avec la mobilité, mais nous avons trouvé des différences notables de composition en acides aminés et en glucides dans les trois fractions que nous avons analysées.

On sait que dans la caséine bovine, il existe un constituant caractéristique, la caséine $\chi$, qui est sensible à la chymosine et à la pepsine, insensible au calcium et qui contient de la cystéine et des glucides, les autres constituants majeurs, $\alpha_{\mathrm{s}}$ et $\beta$ ne possédant pas ces propriétés. Alais et Jollès $(1969,1970)$, ont posé le problème de l'existence d'un constituant de type $\chi$ dans des caséines d'espèces monogastriques. A partir de la caséine humaine, ils ont séparé des fractions fortement enrichies en un constituant très sensible à la chymosine, mais se trouvant dans des zones différentes de mobilités électrophorétiques en milieu alcalin (partie rapide et partie lente). Dans le présent travail, nous avons observé que seule la fraction rapide est sensible à la chymosine et insensible aux ions calcium ; on peut donc penser que la " caséine $\chi$ " humaine se trouverait concentrée dans cette fraction. Malpress et Seid-Akhavan (1966) et Groves et Gordon (1970) ont également observé la sensibilité à la chymosine dans la fraction la plus rapide à l'électrophorèse, mais pour ces deux derniers auteurs, toutes les fractions seraient sensibles au calcium, ce que nous n'avons pas observé.

La détermination des acides aminés dans les trois constituants homogènes à l'électrophorèse à $\mathrm{pH} 8,6$ révèle des ressemblances entre les deux constituants de la fraction rapide et des différences marquées entre ceux-ci et le constituant de mobilité moyenne, ce dernier paraissant être dépourvu de cystéine et étant beaucoup plus riche en proline (caractéristiques de la caséine $\beta$ bovine, de mobilité moyenne).

Des essais de fractionnement de la caséine humaine sont poursuivis à partir de laits individuels, en portant l'attention surtout sur les constituants de faible mobilité électrophorétique. Ils sont accompagnés d'expériences de déphosphorylation et désialylation pour tenter d'expliquer les anomalies observées.

\section{Rés u mé}

L'examen de nombreux échantillons de caséine humaine a révélé une grande variabilité de plusieurs propriétés. Des essais de fractionnement par chromatographie sur colonne d'échangeurs d'ions, par 
filtration moléculaire et par électrophorèse préparative en gel ont permis de séparer trois constituants phosphoglycoprotéiques qui ont été analysés. Un constituant de mobilité élevée à pH 8,6 présente des analogies avec la caséine $\chi$ bovine par la sensibilité à la chymosine, l'insensibilité aux ions calcium et la composition chimique.

\section{S u m mary}

Analyses of several samples of human milk casein have shown a rather high variability for some important properties. Numerous assays of fractionation by chromatography, molecular sieving and preparative electrophoresis allowed the separation of three phosphoglycoprotein components which were analyzed. One component is related to bovine $\chi$ - casein by its behaviour toward chymosin and calcium ions and by its chemical composition.

\section{Remerciement}

Nous remercions le Comité de Lorraine de la Fondation pour la Recherche Médicale Française pour son aide matérielle.

Reçu pour publication le 15 mai 1975.

\section{Bibliographie}

Alais (C.) et Jollès (P.). (1964) - 6 th Intern. Cong. Biochem., New-York, II, 137.

Alais (C.) et Jollès (P.) (1969). - J. Chromatogr., 44, 573-580.

Alais (C.) et Jollès (P.) (1970). - Intern. J. Biochem., 1, nº 5, 546-552.

Alais (C.) et Jollès (P.) (1970). - Arch. Biochem. Biophys., 139, 406-409.

Alais (C.), Mocouot (G.), Nitschmann (H. S.) et Zahler (P.) (1953). - Helv. Chim., Acta, 36, 1955.

Bamann (E.), Novotny (E.) et Rohr (L.) (1948). - Chem. Ber., 81, 438.

Groves (M. L.) et Gordon (W. G.) (1970). - Arch. Biochem. Biophys., 140, 47-51.

Groves (M. L.) et Townend (R. E.) (1970). - Arch. Biochem. Biophys., 139, 406-409.

Malpress (F. H.) et HytTEN (F. H.) (1964). - Biochem. J., 91, 130.

Malpress (F. H.) et Seid-AKHaVAN (M.) (1966). - Biochem. J., 101, 764.

Nagasawa (T.), Ryoki (T.), Kiyosawa (I.) et Kuwahara (K.) (1967). - Arch. Biochem. Biophys., 121, 502-507.

Nagasawa (T.), Kiyosawa (I.) et Kuwahara (K. J.) (1969) - J. Dairy Sci., 53, 136-145.

Nagasawa (T.), Kiyosawa (I.) et Kuwahara (K. J.) (1970). - J. Dairy Sci., 54, 987-993.

Nagasawa (T.), Kiyosawa (I.), Kuwahara (K.), Fukuwatari (Y.) et Suzuki (T.J.) (1971). - J. Dairy Sci., 55, 289-293.

Nagasawa (T.), Kiyosawa (I.), Kuwahara (K.) et Inomata (Y.) (1974). - Agr. Biol. Chem., 38, 117-120.

PiEz (K. A.) et Morris (L.) (1960). - Anal. Biochem., 1, 187.

Rondle (C. J.) et Morgan (W. T. J.) (1955). - Biochem. J., 61, 586.

Schultze (H. E.), Schmidtberger (R.) et Haupt (H.) (1958). - Biochem. Z, 329, $490-507$.

Testud (M.) et Ribadeau-Dumas (B.) (1973). - Biochimie, 55, 1085-1093.

Toyoda (M.) et Yamauchi (K.) (1973). - Agr. Biol. Chem., 37, 783-788.

WAKE (R. G.) et BALDWIN (R. L.) (1961). - Biochimica Biophysica Acta, 47, 225.

WARREN (L. J.) (1959). - J. Biol. Chem., 234, 1971-1975. 